\title{
Selektive Filtration - Eine Sortierung im Magnetfeld
}

\section{Christian Eichholz*, Mathias Stolarski und Hermann Nirschl}

Die Kombination von klassischer Filtration und Sortierprozessen im Magnetfeld ermöglicht eine selektive Separation von magnetischen und unmagnetischen Komponenten bei gleichzeitig überlagerter Flüssigkeitsabtrennung aus dem System. Diese Arbeit zeigt den Einfluss von magnetischer Feldstärke und der Wahl des Filtermediums auf die Selektivität der Trennung eines Modellsystems aus Magnetit und Quarzmehl. Zudem wird mit der mehrstufigen selektiven Filtration eine mögliche Prozessvariante demonstriert.

Schlagwörter: Filtration, Magnetseparation, Selektive Separation

Eingegangen: 05. Mai 2008; akzeptiert: 07. Juli 2008

\section{Einleitung}

Die Magnetseparation wurde bisher hauptsächlich als ein reiner Sortierprozess in der Erzverarbeitung und im Recycling eingesetzt [1]. Im Gegensatz dazu handelt es sich bei der hier vorgestellten Anwendung um ein hybrides Verfahren, bei dem Sortierung und Kuchenfiltration kombiniert werden. Denkbar ist beispielsweise der Einsatz zur Aufreinigung und Verarbeitung magnetischer Pigmente oder in der Bioseparationstechnik.

Ein Schwachpunkt biotechnologischer Prozesse ist die Aufarbeitung der Biosuspension, die momentan bis zu $80 \%$ der Investitionsund Betriebskosten bündelt. Deshalb werden verstärkt alternative Methoden zur Aufbereitung gesucht. Eine Möglichkeit stellt die selektive Magnetseparation unter Verwendung spezieller magnetischer Trägerpartikeln mit einer auf das Zielprodukt abgestimmten Oberflächenfunktionalisierung dar [2]. Das Zielprodukt wird selektiv an so genannte Magnetbeads gebunden, deren Gewinnung dann durch die Abtrennung des magnetischen Trägermaterials erfolgt, beispielsweise analog zum hier vorgestellten Verfahren der selektiven Filtration.

Für die Aufreinigung magnetischer Pigmente liegt der Vorteil vor allem in der Abtrennung der Verunreinigungen bei gleichzeitiger Reduzierung des Flüssigkeitsvolumens. Dadurch können in einer längeren Prozesskette die nachfolgenden Apparate deutlich in der Größe reduziert werden. Im Gegensatz zu anderen Verfahren der Magnetseparation (z. B. HGMS) kann dieses Verfahren auch für hochkonzentrierte Suspensionen genutzt werden. Durch Variation von Feldstärke, Filtrationsdruck und Filtermedium ist das Verfahren an die jeweilige Trennaufgabe flexibel anpassbar. Da für die in dieser Arbeit durchgeführten Untersuchungen bereits sehr geringe Magnetfeldstärken für eine erfolgreiche Trennung ausreichend waren, lässt sich das Verfahren durch Erhöhung der Feldstärke auch auf kleinere oder schwächer magnetische Stoffsysteme anwenden.

\section{Theorie}

Bei der kuchenbildenden Filtration wird ein Feststoff durch ein Filtermedium oder durch bereits darüber aufgebaute Feststoffbrücken zurückgehalten, während die Flüssigkeit z. B. aufgrund eines Gasdifferenzdrucks hindurch strömt. Nach dem Aufbau der Feststoffbrücken sind diese für die eigentliche Filtrationswirkung verantwortlich. Aus diesem Grund ist es möglich, die Porenweiten des Filtermediums zur Reduzierung des Durchströmungswiderstands größer als den mittleren Partikeldurchmesser zu wählen. $\mathrm{Zu}$ Beginn der Filtration kommt es zu einem Feststoffdurchschlag ins Filtrat, der aber durch die Feststoffbrücken unterbunden wird. Um eine schnelle Brückenbildung durch Wechselwirkungen der Partikel untereinander zu gewährleisten, ist eine ausreichend hohe Feststoffkonzentration in der Suspension $\left(c_{\mathrm{v}}>10\right.$ Vol.-\%) Voraussetzung. Im Verlauf der Filtration nimmt die Kuchenbildungskinetik durch den sich aufbauenden Filterkuchen und den somit ansteigenden Durchströmungswiderstand ab. Dieses Verhalten kann mit der Gleichung von Darcy beschrieben werden. Die Strömungskraft auf ein einzelnes Partikel in der Suspension lässt sich nach Stokes mit Gl. (1) beschreiben [3]:

\section{Die Aufarbeitung von Biosuspensio- nen bündelt zurzeit bis zu $80 \%$ der Investitions- und Betriebskosten. Eine alternative Metho- de stellt die selek- tive Magnetsepara- tion unter Verwen- dung spezieller magnetischer Trägerpartikel mit einer auf das Ziel- produkt abgestimm- ten Oberflächen- funktionalisierung dar.}


$F_{\mathrm{D}}=3 \pi \eta_{1} d u$

Nach Abschluss der Filterkuchenbildung kann sich eine Filterkuchenwäsche anschließen. Grundsätzlich wird zwischen Verdrängungs- und Verdünnungswäsche unterschieden [4]. Eine wichtige Größe zur Bewertung des Waschergebnisses bzw. des Waschwasserverbrauchs ist das Waschverhältnis $\delta$. In dieser Arbeit wird es als der Quotient aus Waschfluidmasse $m_{1}$ und anfänglicher Feststoffmasse $m_{\mathrm{s}}$ im Prozessraum definiert:

$\delta=\frac{\text { Waschfluidmasse } m_{1}}{\text { Feststoffmasse } m_{\mathrm{s}}}$

Während bei der Verdrängungswäsche die Waschverhältnisse sehr gering sind, wird bei der Verdünnungswäsche Waschflüssigkeit im Überschuss zugegeben und der Filterkuchen vollständig resuspendiert. Die jeweilige Verunreinigung des Filterkuchens wird als Konzentration bezogen auf die Anfangskonzentration der Verunreinigung angegeben:

netkraft wirkt nur wenn das Magnetfeld einen Gradientenverlauf aufweist.

$c_{\mathrm{i}}^{*}=\frac{c_{\mathrm{i}}}{c_{0}}$

Der Trenngrad eines Waschschrittes $i$ lässt sich somit aus dem Quotienten zweier normierter Konzentrationen berechnen:

$\eta_{\mathrm{i}}=\frac{c_{\mathrm{i}}^{*}}{c_{\mathrm{i}-1}^{*}}$

Bei einer idealen mehrstufigen Wäsche mit konstantem Trenngrad pro Schritt kann der Verlauf der Restverunreinigung aus dem Trenngrad des ersten Waschschrittes und den Waschfluidmassen bestimmt werden:

$\eta_{\mathrm{i}}=\eta_{1}^{\frac{\delta_{\mathrm{i}}}{\delta_{1}}}$

Entsprechend ihrer magnetischen Eigenschaften lassen sich dia-, para- und ferromagnetische Materialien unterscheiden. Die $\mathrm{Zu}$ ordnung erfolgt aufgrund der volumetrischen Suszeptibilität $\chi$, die das Verhalten des jeweiligen Materials in einem magnetischen Feld beschreibt. Diamagnetische Effekte treten erst bei sehr starken Magnetfeldern erkennbar in Erscheinung. Daher werden Materialen, die rein diamagnetische Eigenschaften besitzen im Allgemeinen als unmagnetisch bezeichnet. Im Gegensatz dazu verstärken ferromagnetische Materialien $(\chi>>0)$ lokal das externe Feld und erfahren eine Kraft in Richtung des stärker werdenden externen Feldes. Abhängig von der Suszeptibilität und der Magnetfeldstärke wird das Material bis zu einer bestimmten Magnetisierung $M$ magnetisiert. Die resultierende Magnetkraft auf ein Partikel kann als Funktion der Magnetisierung, des Partikelvo- lumens und des Gradienten des externen Feldes $\nabla B$ dargestellt werden:

$F_{M}=V \rho M \nabla B$

Eine externe Magnetkraft wirkt demnach nur, wenn das Magnetfeld einen Gradientenverlauf aufweist. Aber auch in einem homogenen Magnetfeld sind magnetische Effekte zu beobachten. Aufgrund magnetischer interpartikulärer Wechselwirkungen ziehen sich benachbarte Partikel in Magnetfeldrichtung an, während sie sich senkrecht dazu abstoßen. Als Konsequenz orientieren sich die Partikel in kettenförmigen Agglomeraten [5].

In einem magnetfeldüberlagerten Filtrationsprozess ermöglicht die externe Magnetkraft, Fluid- und Feststoffbewegung zu entkoppeln. Dadurch wird ein zusätzlicher Freiheitsgrad gewonnen, mit dem sich der Filterkuchenaufbau in der Filterkammer steuern lässt. Bei entsprechender Magnetfeldkonfiguration wird der Kuchenaufbau verzögert bzw. bei Überschreitung einer kritischen Feldstärke zu Beginn vollständig unterbunden, sodass das Filtrat nahezu ohne Widerstand durch das Filtermedium strömen kann [6, 7].

\section{Methoden und Materialien}

Für die Vorversuche zur Auswahl eines geeigneten Filtermediums und zur Untersuchung des Einflusses der magnetischen Feldstärke diente ein Kunststofffilter mit einer Filterfläche von $10 \mathrm{~cm}^{2}$ (CUNO-Filter Tri-47, CUNO Filtration). Im Anschluss stand mit einer gerührten Drucknutsche $\left(A=50 \mathrm{~cm}^{2}\right.$, Bokela $\mathrm{GmbH}$ ) ein weiterer Versuchsapparat für die mehrstufige selektive Separation zur Verfügung. Es wurde ein Elektromagnet (Steinert Elektromagnetbau $\mathrm{GmbH}$ ) verwendet, in dessen zentrale Bohrung die jeweilige Nutsche eingesetzt wurde. Die untersuchten Magnetfeldstärken betrugen $0 \mathrm{~T}, 0,05 \mathrm{~T}, 0,11 \mathrm{~T}$ und 0,22 T. Die externe Magnetkraft wirkte dabei entgegengesetzt zur Filtrationsrichtung; der Filtrationsdruck betrug 0,2 bar und 0,8 bar. Als Filtermedien wurden sonderkalandrierte (SK) Filtertücher mit Maschenweiten von 20, 68 und $80 \mu \mathrm{m}$ gewählt.

Zusätzlich zum axialen Feldgradienten, der eine Partikelwanderung weg vom Filtermedium verursacht, wirkt bei der verwendeten Magnetspule ein radialer Gradient, d.h. dass magnetische Partikeln auch eine Kraft in Richtung des Nutschenrands erfahren. Im CUNOFilter erzwang ein auf die Suspension aufgesetzter Presskolben eine reguläre Kuchenbildung. Bei der mehrstufigen Filtration wurde 
auf einen Presskolben verzichtet. Die magnetischen Partikel lagerten sich am Rand der Nutsche $a b$, waren aber mit Hilfe des Rührers einfach $\mathrm{zu}$ resuspendieren. Dazu wurde nach jedem Filtrationsschritt ca. $200 \mathrm{~mL}$ Waschwasser zugeführt und $5 \mathrm{~min}$ mit $275 \mathrm{U} / \mathrm{min}$ ohne überlagertes Magnetfeld gerührt.

Als Modellkomponenten kamen ein ferromagnetisches Schwarzpigment (natürliches Magnetit, $\mathrm{Fe}_{3} \mathrm{O}_{4}, d_{50}=15 \mu \mathrm{m}, \rho=5 \mathrm{~g} / \mathrm{cm}^{3}$ ) und ein Quarzsand (SF800, $d_{50}=2 \mu \mathrm{m}, \rho=2,65 \mathrm{~g} / \mathrm{cm}^{3}$ ) zum Einsatz. Beide Komponenten wiesen eine gewisse Breite in der Partikelgrößenverteilung auf. Die Feststoffkonzentration je Komponente in der Suspension betrug $c_{v}=10 \%$.

\section{Ergebnisse und Diskussion}

Es wurden Filtrationsversuche mit verschiedenen Filtermedien und Feldstärken durchgeführt, um den jeweiligen Einfluss auf die selektive Trennung zu charakterisieren. Die Untersuchungen begannen mit einem Filtermedium mit einer Porenweite von $20 \mu \mathrm{m}$. Bei einer herkömmlichen Filtration sedimentiert das Eisenoxid aufgrund der höheren Dichte und des größeren Partikeldurchmessers schneller und bildet eine abgegrenzte Schicht im unteren Teil des Filterkuchens. Der Einfluss des im nächsten Schritt überlagerten Magnetfelds ist an der umgekehrten Schichtung des Filterkuchens zu erkennen (Abb. 1). Beide Feststofflomponenten tragen zur Bildung des Filterkuchens bei; lediglich wenige Quarzpartikeln gelangen bei der Überlagerung des magnetischen Feldes als Trübstoß in das Filtrat (Abb. 2a).

Durch die Wahl eines Filtermediums mit größerer Maschenweite (hier: $68 \mu \mathrm{m}$ ) ist es möglich, die Feststoffe während des Filtrationsprozess voneinander $\mathrm{zu}$ separieren. In diesem Fall trägt hauptsächlich das Magnetit zur Filterkuchenbildung bei (Abb. 2b), während sich der Volumenanteil des Quarzes im Filterkuchen von $x_{\mathrm{v}}=49 \%$ auf $x_{\mathrm{v}}=27 \%$ verringert ( $=17 \%$ Gesamtvolumenanteil in Abb. $2 b$ ).

Bei der Verwendung des Filtermediums SK80 mit einer Maschenweite von $80 \mu \mathrm{m}$ verschlechtert sich hingegen die Reinheit des abgetrennten Quarzmehls bei nahezu gleich bleibender Filtrationsgeschwindigkeit durch den erhöhten Anteil an Magnetit, der als Trübstoß in das Filtrat gelangt (Abb. 2c). Dies weist darauf hin, dass zuvor die Ablagerung einer bestimmten Partikel- bzw. Agglomeratfraktion des Magnetits auf dem Filtermedium SK68 bei 0,11 T nicht verhindert wurde. Bei genügend großer Maschenweite werden diese Partikel jedoch nicht zurückgehalten und gelangen in

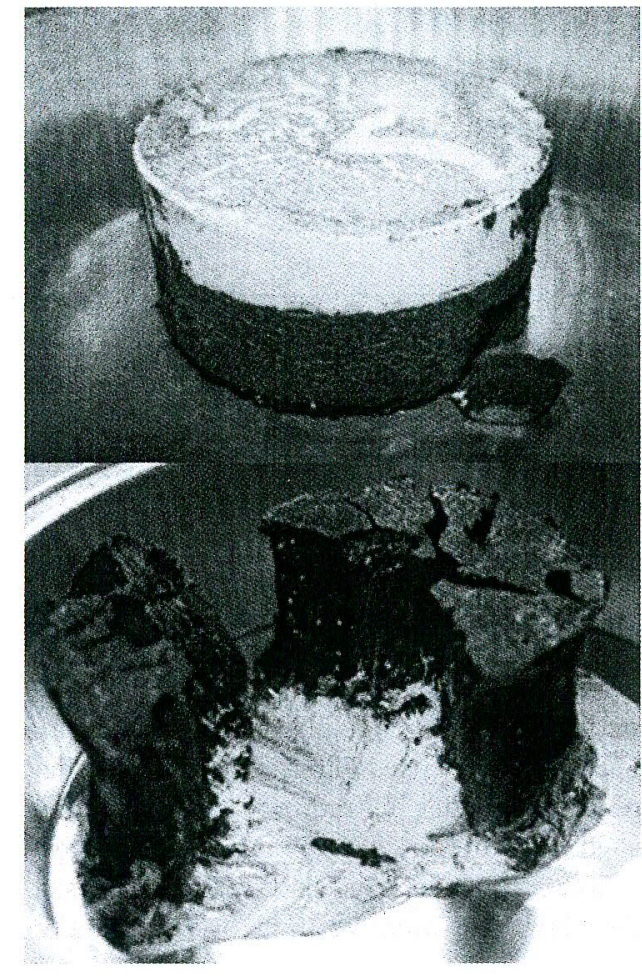

das Filtrat. Gleichzeitig erhöht sich auch der Quarzanteil im Filtrat, da das Filtertuch weniger blockiert ist. Der Anstieg der Quarzmenge im Filtrat bei Erhöhung der Feldstärke (Abbn. 2d - f) lässt sich durch den gleichen Ansatz erllären. Blockieren bei geringer Feldstärke magnetische Partikeln und Agglomerate noch teilweise das Filtermedium, so werden diese bei stärkeren Magnetfeldern vom Filtermedium ferngehalten und es kann mehr Quarz mit der Flüssigkeit zusammen abströmen.

Zusätzlich wirkt sich das Magnetfeld auch auf die Filtrationsgeschwindigkeit aus. In Abb. 3 sind die Filtratanfälle einer klassischen
Abbildung 1. Filterkuchen des Zweikomponentensystems (CUNO-Filter): ohne Magnetfeld (oben), mit Magnetfeld (unten).

Bei einer herkömmlichen Filtration sedimentiert das Eisenoxid aufgrund der höheren Dichte und des größeren Partikeldurchmessers schneller und bildet eine abgegrenzte Schicht im unteren Teil des Filterkuchens.

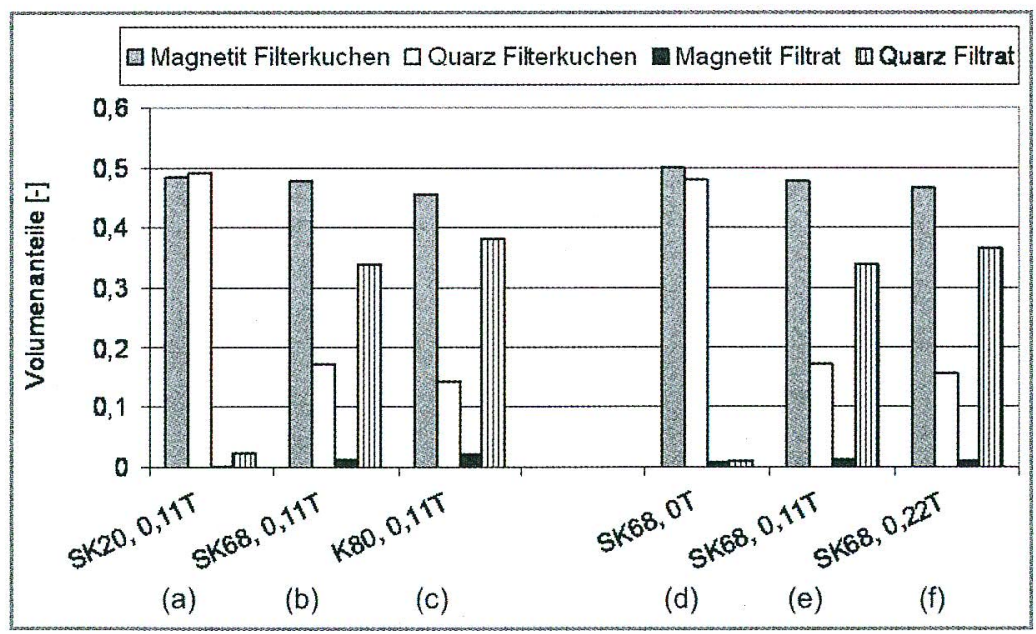

Abbildung 2. Gesamtvolumenanteile von Magnetit und Quarz in Filterkuchen und Filtrat in Abhängigkeit der Magnetfeldstärke und des Filtermediums. 
Abbildung 3. Filtratanfall bei klassischer und magnetfeldüberlagerter Filtration.

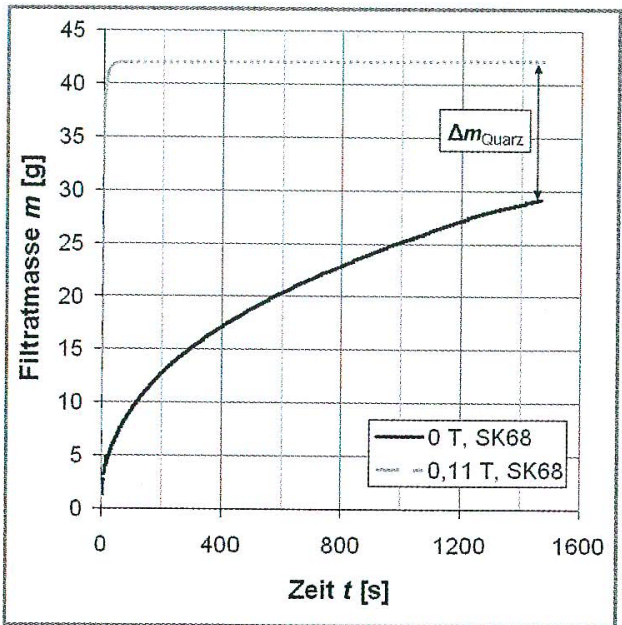

und einer magnetfeldüberlagerten Filtration bei Verwendung des Filtermediums SK68 dargestellt. Bei der Filtration ohne Magnetfeld stellt sich die bekannte Kinetik ein. Mit fortschreitender Versuchsdauer verlangsamt sich der Filtratfluss, weil der anwachsende Filterkuchen den Filtrationswiderstand erhöht. Im Gegensatz dazu läuft der Prozess mit überlagertem Magnetfeld schlagartig ab, da die gewählte Feldstärke größer ist als die kritische Feldstärke, bei der die Filterkuchenbildung unterbunden wird. Filtrat und Quarzpartikel treten ungehindert durch das Filtermedium hindurch. Die Filtrationsdauer konnte in einem Zyklus um den Faktor 100 verringert werden. Der Unterschied in den maximalen Filtratmassen lässt sich durch den erhöhten Feststoffanteil im Filtrat erklären.

Das Ergebnis einer mehrstufigen selektiven Filtration mit sechs Zyklen in der gerührten Drucknutsche ist in Abb. 4 dargestellt. Im Gegensatz zu den Vorversuchen wurde mit geringerer Feldstärke $(0,05 \mathrm{~T})$ und Filtrationsdruck (0,2 bar) gearbeitet. Das Verhältnis $F_{\mathrm{M}}: F_{\mathrm{D}}$ (Gl. (6):Gl. (1)) wurde dabei jedoch ähnlich zu den Versuchen mit 0,11 T gehalten, um gleiche Trennbedingungen $\mathrm{zu}$ garantieren. Als Filter-

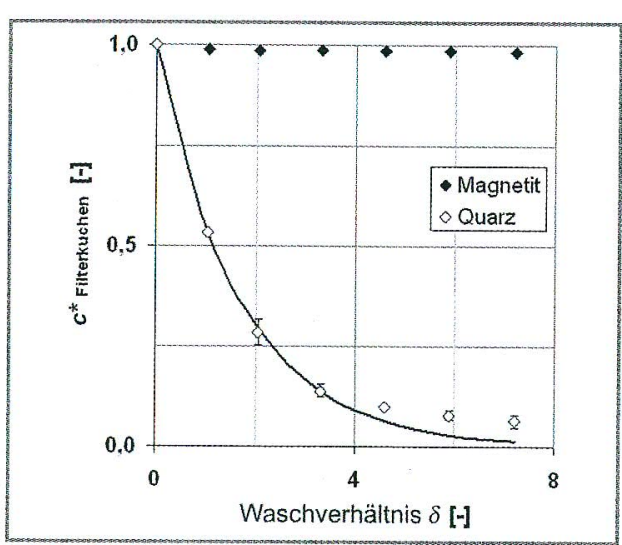

medium war ebenfalls das SK68 gewählt. Zu Versuchsbeginn liegen beide Komponenten mit der gleichen Volumenkonzentration vor, nach den sechs Zyklen hat sich der Quarzanteil im Filterkuchen von $50 \%$ auf $6 \%$ reduziert, während der Anteil des Magnetits im Filtrat lediglich $1 \%$ beträgt.

An dem Verlauf der normierten Konzentrationen von Magnetit und Quarz in Filterkuchen ist ebenfalls deutlich zu erkennen, dass das Quarzmehl aus dem Verfahrensraum ausgewaschen wird, während das Eisenoxid nahezu vollständig in der Nutsche verbleibt (Abb. 4).

Schon nach wenigen Schritten nähert sich der Quarzanteil im Filterkuchen dem Plateau der Restverunreinigung an. Dies liegt zum einen an den Wechselwirkungen zwischen den Partikeln, die dazu führen, dass sich ein Teil des Quarzes zwischen den Magnetitpartikeln einlagert. Zum anderen werden größere Quarzpartikel vom Filtermedium zurückgehalten. Bestätigt wird dies durch den Vergleich mit dem berechneten Verlauf der idealen Wäsche (Gl. (5)). Der konstante Trenngrad lässt sich aus dem ersten Trennschritt ermitteln, der mit einem relativen Fehler von $0,7 \%$ reproduzierbar ist. $\mathrm{Ab}$ einem Waschverhältnis $\delta>4$ liegt die Kurve unterhalb der experimentellen Werte, d.h. das sich bereits an diesem Punkt durch die zuvor genannten Effekte der reale Trenngrad im Vergleich zum ersten Schritt verschlechtert hat. Das mehrstufige Verfahren verliert also mit hohem Waschverhältnis deutlich an Effizienz.

Das Verfahren ist mit einer herkömmlichen Verdünnungswäsche vergleichbar, bei der allerdings eine wesentlich bessere Abtrennung der verunreinigten Waschflüssigkeit erzielt wird, da diese direkt abströmen kann, ohne den Filterkuchen wieder $\mathrm{zu}$ verunreinigen. Auf gleiche Weise können auch bei der Umsetzung der eingangs erwähnten Bioseparation die beladenen magnetischen Trägerpartikeln von der Fermentationsbrühe getrennt werden.

\section{Schlussfolgerung}

Es konnte gezeigt werden, dass die Kombination von klassischer Filtration und magnetischer Sortierung $\mathrm{zu}$ einer Trennung verschiedener Materialen bei gleichzeitiger Entwässerung des Filterkuchens führt. Beide eingesetzten Komponenten liegen nach der Trennung in hoher Reinheit vor, gleichzeitig ist ein deutlicher Kinetikgewinn bei der Filtration zu verzeichnen. Das Filtermedium verhindert dabei einen größeren Durchschlag der magnetischen Komponente in das Filtrat. Für ein optimales Trennergebnis ist allerdings die
Abbildung 4. Restverunreinigung des Filterkuchens in Abhängigkeit des Waschverhältnisses (experimentell und berechnet). 
Wahl eines auf das Stoffsystem abgestimmten Filtermediums notwendig. Diese Untersuchung ist ein wichtiger Schritt auch in Richtung der Anwendung dieses Prinzips in der selektiven Bioseparation.

Diese Arbeit entstand im Rahmen eines Forschungsprojekts zur selektiven Mag. netfiltration mit dem Forschungszentrum Karlsruhe, der Bokela GmbH, Karlsruhe, und der Steinert Elektromagnetbau $\mathrm{GmbH}$, Köln. Die Autoren bedanken sich für die Projektförderung durch das BMWi.

\section{Dipl.-Ing. C. Eichholz}

(christian.eichholz@mvm.uni-karlsruhe.de), Dipl.-Ing. M. Stolarski,

\section{Prof. Dr.-Ing. H. Nirschl,}

Institut für Mechanische Verfahrenstechnik und Mechanik, Universität Karlsruhe (TH), Germany.

\section{Formelzeichen}

B $[\mathrm{T}] \quad$ magnetische Flussdichte

c [-] Konzentration

d $[\mathrm{m}] \quad$ Partikeldurchmesser

$F \quad[\mathrm{~N}] \quad$ Kraft

$M \quad\left[\mathrm{~A} \mathrm{~m}^{2} \mathrm{~kg}^{-1}\right]$ Magnetisierung

$m \quad[\mathrm{~kg}] \quad$ Masse

$t \quad[s] \quad$ Zeit

$u \quad\left[\mathrm{~m} \mathrm{~s}^{-1}\right] \quad$ Geschwindigkeit

$\begin{array}{ll}V & \left.\mathrm{~m}^{3}\right] \quad \text { Volumen }\end{array}$

$\begin{array}{lll}\delta & {[-]} & \text { Waschverhältnis } \\ \eta & {[-]} & \text { Trenngrad } \\ \eta & {[\text { Pa s] }} & \text { Viskosität } \\ \chi & {[1]} & \begin{array}{l}\text { volumetrische Suszeptibi- } \\ \text { lität }\end{array} \\ \rho & {\left[\mathrm{kg} \mathrm{m}^{-3}\right]} & \text { Dichte } \\ \mathrm{x} & {[-]} & \text { Anteil }\end{array}$

\section{Indices}

0 Ausgangszustand

50 Mittelwert

D Strömung

i Teilschritt

1 liquid

M Magnet

s solid

V Volumen

\section{Literatur}

[1] J. Svoboda, Magnetic Methods for the Treatment of Minerals, in Developments in Mineral Processing, Vol. 8, Elsevier Sc. Publishers, Amsterdam 1987.

[2] I. Safarik, L. Ptackova, M. Safarikova, Biotechnol. Lett. 2001, 23, 1953.

[3] M. Stieß, Mechanische Verfahrenstechnik, 2. ed., Band 1 und 2, Springer, Berlin 1995.

[4] F. Ruslim, J. Fleischer, H. Nirschl, U. Peuker, Chem. Ing. Tech. 2006, 78 (6), 715. DOI: 10.1002/cite. 200600011

[5] S. Charles, Chem. Eng. Commun. 1988, 67, 145.

[6] B. Fuchs, M. Stolarski, W. Stahl, H. Nirschl, Filt. J. 2006, 6 (4), 333.

[7] C. Eichholz, M. Stolarski, V. Goertz, H. Nirschl, Chem. Eng. Sci. 2008, 63, 3193. 